\title{
Unraveling the clonal hierarchy of somatic genomic aberrations
}

\author{
Davide Prandi ${ }^{1}$, Sylvan C Baca ${ }^{2,3,4}$, Alessandro Romanel ${ }^{1}$, Christopher E Barbieri ${ }^{5,6,8}$, Juan-Miguel Mosquera ${ }^{6,8}$, \\ Jacqueline Fontugne ${ }^{6}$, Himisha Beltran ${ }^{7,8}$, Andrea Sboner ${ }^{6,8,9}$, Levi A Garraway ${ }^{2,3,4}$, Mark A Rubin ${ }^{5,6,8}$ \\ and Francesca Demichelis ${ }^{1,8,9^{*}}$
}

\begin{abstract}
Defining the chronology of molecular alterations may identify milestones in carcinogenesis. To unravel the temporal evolution of aberrations from clinical tumors, we developed CLONET, which upon estimation of tumor admixture and ploidy infers the clonal hierarchy of genomic aberrations. Comparative analysis across 100 sequenced genomes from prostate, melanoma, and lung cancers established diverse evolutionary hierarchies, demonstrating the early disruption of tumor-specific pathways. The analyses highlight the diversity of clonal evolution within and across tumor types that might be informative for risk stratification and patient selection for targeted therapies. CLONET addresses heterogeneous clinical samples seen in the setting of precision medicine.
\end{abstract}

\section{Background}

Cancer arises from initiating cells (clones) that undergo intense evolutionary selection during disease progression and can be widely altered during treatment. The tumor cell evolutionary process may lead to subclonal divergence resulting in genetic and molecular heterogeneity. Computational approaches to establish maps of cancer evolution might inform clinical risk stratification and treatment strategies. Analysis strategies have been developed to address tumor DNA purity and cancer cell ploidy, but there remains a gap for the analysis of minimally aberrant or highly heterogeneous tumors.

Over the past years, several methods have been developed to quantify DNA admixture and ploidy from high density single-nucleotide polymorphism (SNP) array data [1-4] that utilize the relative abundance of specific allele signal (B allele frequency (BAF)) and the tumor over normal signal ratio (referred to as $\log R$ ) to measure the complexity of the underlying cellular population. Global optimization methods are applied to find the configuration of DNA admixture and ploidy that better

\footnotetext{
* Correspondence: demichelis@science.unitn.it

${ }^{1}$ Centre for Integrative Biology, University of Trento, Povo Trento 38123, Italy ${ }^{8}$ Institute for Precision Medicine, Weill Medical College of Cornell University and New York Presbyterian Hospital, New York, NY 10065, USA

${ }^{9} \mathrm{HRH}$ Prince Alwaleed Bin Talal Bin Abdulaziz Alsaud Institute for Computational Biomedicine, Weill Medical College of Cornell University, New York, NY 10065, USA
} Full list of author information is available at the end of the article account for the observed values of BAF and Log R. More recent tools [5-9] exploit the rich statistical properties of massively parallel sequencing to provide base-pair data resolution. Using germline heterozygous SNP loci (hereafter called informative SNPs), tumor purity and ploidy are estimated analyzing allelic fraction $(\mathrm{AF})$ values (that is, the fraction of sequencing reads supporting the reference base) in a way resembling the use of BAF data in SNP arrays. Subclonal alterations will appear as outliers from the computed admixture and ploidy.

All these tools apply a global approach (that is, the AF (or BAF) values are all thrown into an inference algorithm that eventually returns DNA purity and ploidy). Global methods are well-suited for tumor samples with fairly homogenous genomic aberrations (high ratio of clonal versus subclonal lesions). In the clinical setting, where tumor samples might exhibit heterogeneity due to progression or subsequent to multiple lines of treatment, and for tumor types that undergo structural changes such as 'chromoplexy' events in prostate cancer (that is, abundant DNA rearrangements and deletions that arise in a highly interdependent manner) [10], these approaches may prove suboptimal as they ignore the genomic diversity.

These observations prompted us to develop a second generation tool based on local (in contrast to global) optimization where estimates of purity and ploidy are derived from few clonal events (Figure 1). We noted that the AF 


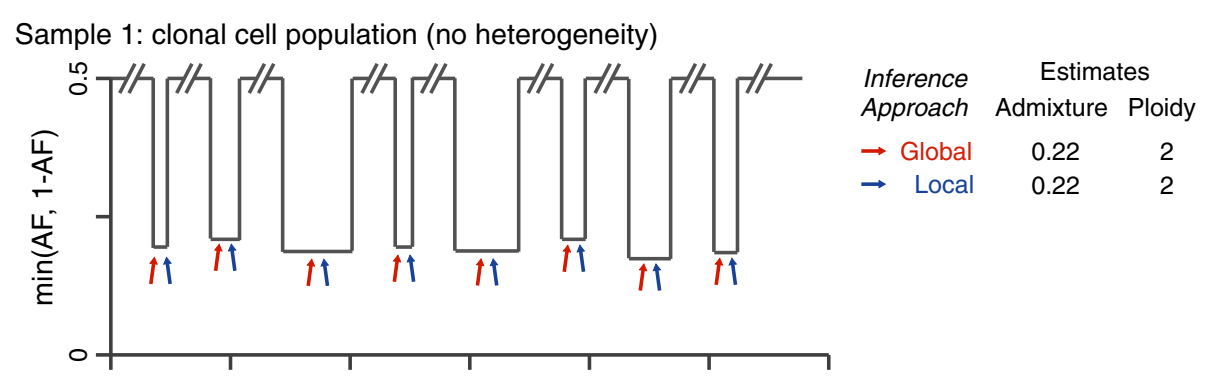

Sample 2: multiclonal cell populations (high heterogeneity)

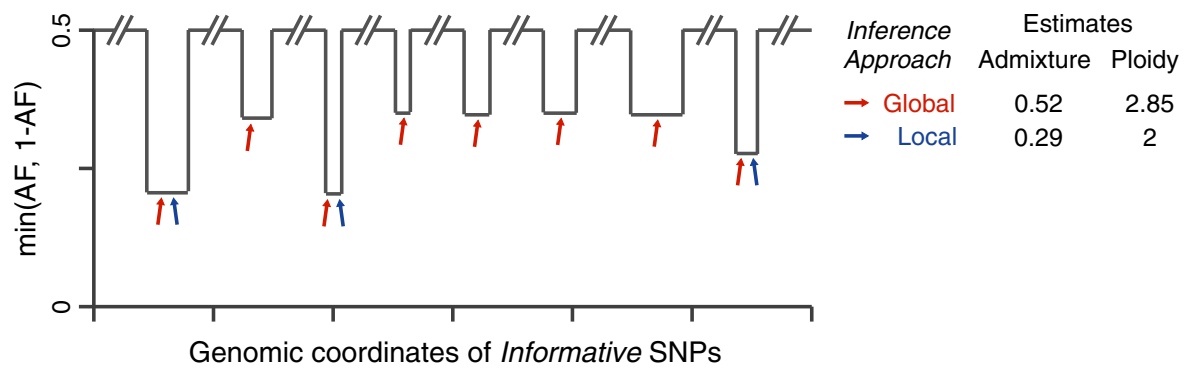

Figure 1 Local versus global approaches to clonality analysis. The local optimization approach to compute global admixture (1 - purity) and ploidy is schematically explained and compared with a global optimization approach on two cancer samples with different levels of lesion heterogeneity. Copy number neutral regions show nominal AF of 0.5; AFs of mono-allelic deletions depend on lesion clonality and on global admixture. Arrows point to lesions used by global (red) or local (blue) approach. For sample 1 showing a profile compatible with clonal cell population (that is, each lesion demonstrates approximately the same AF value), the two approaches return equivalent estimates. For sample 2 (high heterogeneity) the local approach focuses on lesions with the highest level of clonality (blue arrows), resulting in a realistic estimate of the sample admixture.

values of the informative SNPs within a somatic deletion result from the composition of signal from three cell populations: (i) non-tumor cells (contributing to the DNA admixture); (ii) tumor cells without the deletion; and (iii) tumor cells harboring the deletion (that is, a subclonal deletion given (ii) and (iii)). By modeling the probability distribution of the observed AFs, we compute a local estimate of the DNA admixture (1 - DNA purity) that accounts for both normal cell admixture and subclonal tumor cell population. After estimating local admixture values for all deletions across the genome, only selected lesions (from the most clonal side of the spectrum) contribute to the computation of the tumor sample global admixture. In the presence of homogenously aberrant genomes (Figure 1, top), global and local approaches result in similar estimates; for heterogeneous genomes (Figure 1, bottom), the local approach focused on selected lesions (blue arrows in Figure 1) leads to more realistic estimates. Here, we present the full implementation of CLONET (CLONality Estimate in Tumors) and study the clonality of somatic aberrations from whole genome sequencing (WGS) data across 3 tumor types comprising 55 individuals with primary prostate cancer [10], 24 metastatic melanomas [11], and 21 lung adenocarcinomas [12].

\section{Results}

Clonality assessment of aberrations from sequencing reads We reasoned that the reads mapped into a genomic window can be partitioned in two sets: one set includes reads that equally represent parental chromosomes (copy number neutral reads); and the other set contains reads from only one parent chromosome (active reads). There are four main steps that, starting from neutral read counts, allow inference of clonality of any genomic window. First, we estimate the percentage of neutral reads within a genomic segment independently of its $\log R$ value. Second, we use the $\log R$ value to relate the neutral reads percentage with a local estimate of DNA admixture. Local estimates are then aggregated to estimate global admixture and clonality of somatic copy number aberrations (SCNAs). Third, aneuploidy genomes are identified and the analysis corrected accordingly. Finally, we extend the analysis to point mutations (PMs) and structural rearrangements (REARRs) in a coherent manner. In the following we will briefly detail each step.

For each genomic segment Seg, the expected AF of the informative SNP in Seg has bimodal distribution that relates to the composition of the DNA sample; the distance between the two modes is proportional to the percentage of neutral reads $\beta$ (see Materials and methods). The expected distribution of the AF (Figure $2 \mathrm{~A}$ ) varies accordingly with $\beta$ and $N_{\text {ref }}$, which is the proportion of reference base reads in the allele represented by active reads. For each input segment Seg, optimization based on swarm intelligence [13] finds a $\beta$ that minimizes the difference between the expected and the observed AF distribution (see Materials and methods). Then, the Log R of Seg allows computing a local estimate of the admixture. If $\mathrm{Seg}$ 


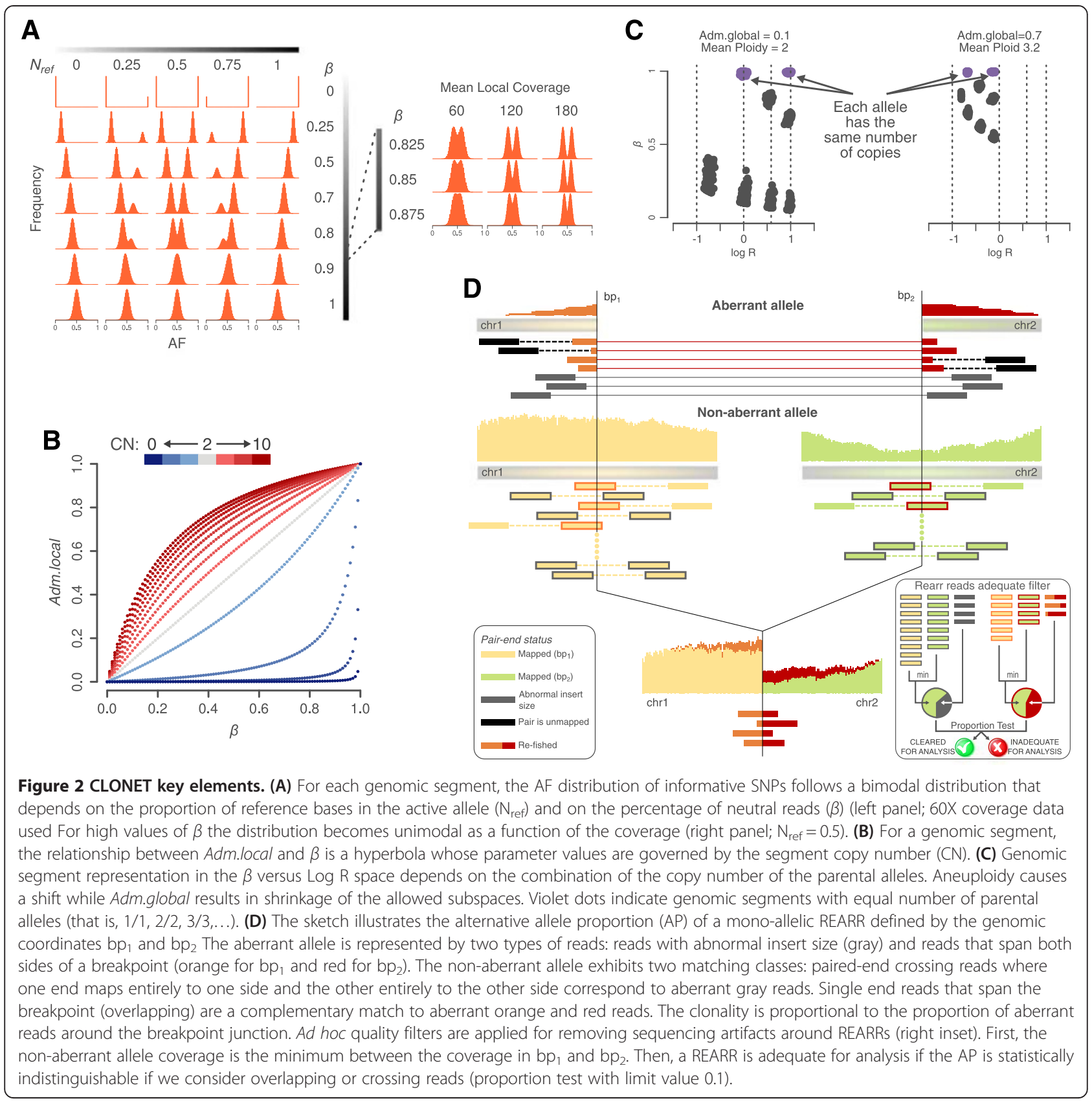

defines a mono-allelic deletion, $\beta$ corresponds to the percentage of reads deriving from cells that do not harbor the deletion (see Materials and methods) and relates to a local estimate of the percentage of admixed cells, Adm.local, as (proof in Additional file 1):

$$
\text { Adm.local }=\frac{\beta}{2-\beta}
$$

Reasoning that the signal from normal cells is uniform along the genome, local admixture values are then clustered, and the lowest median value among all the clusters determines the global admixture (Adm.global) of the sample. Reasoning that the more the local admixture value differs from the global one the more $\mathrm{Seg}$ is subclonal, the clonality of $\mathrm{Seg}, \mathrm{Cl}_{\mathrm{Seg}}$, is computed as the percentage of tumor cells in a sample harboring Seg. If Seg is a gain, Equation 1 extends by rescaling the percentage of neutral reads $\beta$ to recover the percentage of reads sequenced from cells that does not harbor the gain of Seg (Figure 2B; Materials and methods). Bi-allelic deletions are treated separately; if the deletion is clonal, its AF distribution has binomial distribution $(\beta=1)$ and represents only DNA admixture, but in case of subclonality, the value of 
$\beta$ is proportional to the percentage of tumor cells that do not harbor the deletion (Additional file 2).

Aneuploidy causes a shift in the $\log \mathrm{R}$ versus $\beta$ space of a sample (Figure 2C). In any genomic segment with an empty active reads set (neutral copy number segment) each allele has the same number of copies, and its $\beta$ is 1 by definition. The ploidy of a sample is the shift in the $\log R$ values of the neutral segment that best accounts for the observed $\log \mathrm{R}$ values. Log $\mathrm{R}$ data are then corrected for both ploidy and Adm.global to achieve better estimates of the segment copy number (Figure S2A-C in Additional file 3).

Clonality estimates of PMs build on the assumption that reads supporting the alternative allele are representative of the amount of tumor DNA harboring the mutation. In particular, the proportion of reads supporting the alternative allele (AP) of a 100\% pure and clonal hemizygous PM has symmetric binomial distribution. The Adm.global value represents the percentage of reads from admixed cells that have to be ignored to compute the correct value of the AP. A PM is subclonal when its corrected AP has a low probability to be clonal (type I error $<0.05$ ). The same principle applies to REARRs by properly selecting reads from rearranged tumor cells. The total number of reads that span both sides of a breakpoint defining a REARR [14] is a proxy of the number of cells harboring the rearrangement (Figure 2D). After removing reads representative of global admixture, the difference between the expected and the observed proportion of reads supporting the alternative allele is proportional to the subclonality of the considered REARR (Additional file 1).

\section{Inferring the order of mutations in a tumor sample}

The assessment of the clonality of each somatic aberration enables the deconvolution of the sequence of oncogenic events that occur during tumor initiation or progression. Assuming that clonal alterations originated prior to subclonal alterations within the same tumor, we examined pairs of genes that are aberrant in the same sample and across multiple tumors to determine the directionality of the clonal-subclonal hierarchy. However, different error sources may introduce a bias into the distribution of the AF that could lead to inaccurate clonality estimates. To minimize the number of false positives (clonal aberrations called subclonal), we compute the estimation uncertainty around $\beta$ (Figure 3A) and propagate it to clonality values (see Materials and methods). Error management enables robust comparison of aberration clonality across different tumor sample data. We then apply the following algorithm to determine the progression on somatic aberrations: if a clonal aberration $\mathrm{A}_{1}$ and a subclonal aberration $\mathrm{A}_{2}$ cooccur within the same sample $S$, we assume that $A_{1}$ has been acquired before $A_{2}$ in $S$ and we say that $A_{1}$ precedes $A_{2}$ in $S$. To then derive the rule that links aberration $A_{1}$ and $A_{2}$, the same dependency has to be found consistently across many samples. This strategy can produce an evolution path draft (that is, a pictorial representation of the potential temporal relations among somatic aberrations observed in a sample set (Figure 3B)). In the presence of adequate sample size and frequencies of cooccurring aberrations, the statistical significance of the relation between $A_{1}$ and $A_{2}$ can be assessed by testing the null hypothesis that two aberrations are independent (that is, $A_{1}$ precedes $A_{2}$ or $A_{2}$ precedes $A_{1}$ are equally likely) and consider a binomial distribution $B(n, p)$ with number of trials $n$ equals the number of samples where $A_{1}$ is clonal and $A_{2}$ is subclonal or vice versa, and success probability $P=0.5$ (binomial test with $5 \%$ significance would require a minimum of 6 out of 6 samples where $A_{1}$ precedes $\mathrm{A}_{2}$ ).

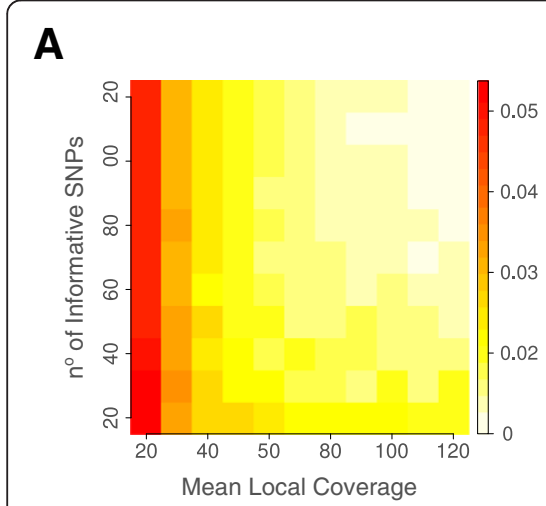

B

Figure 3 Building the evolution path. (A) For each genomic segment, the uncertainty value around $\beta$ is a function of the number of informative SNPs and of the mean local coverage. (B) Tumor evolution paths are built from ordered aberrations consistently observed in multiple samples. On the left, arrows indicate ordered aberrations based on clonality estimates within the same tumor; three distinct aberrations (orange, green, and blue) precede a fourth one (red). Ordered aberrations that find statistical support (as recurrent in multiple samples) contribute to the tumor evolution path (right). 


\section{In silico and in situ experimental validation}

To assess if the coverage depth typical of large scale sequencing experiments (around or below 100X for WGS) has an effect on clonality estimates of SCNAs or PMs, we first queried MiSeq ultra-deep sequencing (approximately $65,000 \mathrm{X}$ ) data generated from a set of 18 aberrant genes from 7 tumor samples analyzed in this study and observed excellent agreement in downstream clonality calls for deletions (Cochran test, $P=1$ ) [10]. Notably, CLONET did not assign clonality values to aberrations in which MiSeq does not confirm WGS-based AP values. Next, we focused on a set of study PMs and assessed high correlation of AP values between WGS and MiSeq (mean coverage $>143,000 \mathrm{X}$ ) data (Figure S3A in Additional file 4; Table S1 in Additional file 5) (Pearson's $r=0.73, P<10 \mathrm{e}-3$ ), suggesting altogether that the study coverage does not significantly impact the ability to assess aberration clonality.

In order to validate the clonality status of more complex structural genomic aberrations, specifically gene rearrangements and homozygous deletions, we turned to in situ tests on human tumor samples. As proof of principle, we first demonstrated the ability to assess rearrangement clonality focusing on well-characterized prostate-specific clonal REARRs involving ERG and TMPRSS2 [15]. Separate clonality analyses of both TMPRSS2-ERG REARRs and of the accompanying $3 \mathrm{Mb}$ interstitial deletion [16] in two prostate tumors (P03-2345 and PR-09-146) demonstrated perfect agreement (Figure 4A; Figure S3B in Additional file 4). Next, we validated by fluoresce in situ hybridization (FISH) analyses two subclonal REARR calls, involving a genomic area $86 \mathrm{~kb}$ upstream of MSR1 (8p22) in patient PR-2525 (Figure 4B) and SPRY2 (13q31.1) in patient PR3042 (Figure S4A in Additional file 6).

Finally, we validated in situ a homozygous deletion along 5q spanning CHD1 that was predicted to be subclonal. Figure $4 \mathrm{C}$ shows the sequencing data information utilized by CLONET to infer the subclonal bi-allelic deletion in patient sample PR-2741 as confirmed by FISH (Figure 4D). The prediction highlights a relatively small subclonal bi-allelic deletion (Figure 4C, region 2) within a larger clonal mono-allelic deletion (Figure 4C, regions 1 and 3), suggesting that selective evolutionary pressure is acting on the genomic region. Patient PR-2525 demonstrated a similar subclonality pattern at the CHD1 locus (Figure S4B in Additional file 6).

\section{Comparison of CLONET and ABSOLUTE}

We compared Adm.global predictions for prostate cancer and melanoma samples with calls reported in the original manuscripts $[10,11]$ (data for lung not publicly available) using a widely used computational method, ABSOLUTE [5], that implements a global estimation approach. Overall agreement was observed (Figure S5A, $\mathrm{B}$ in Additional file 7) in both datasets (Pearson's $\mathrm{r} \geq 0.8$,
$P<10 \mathrm{e}-5)$. Not surprisingly, CLONET allowed for larger fractions of admixture calls compared with ABSOLUTE ( $98 \%$ versus $74 \%$ in prostate and $92 \%$ versus $88 \%$ in melanoma) as the local approach better handles samples with low ratios of clonal to subclonal SCNAs. The average ratio across cases only handled by CLONET is 1.73 versus average ratios across cases handled by both of 5.72 and 6.88 for prostate and melanoma samples, respectively. We further tested CLONET performance on whole exome sequencing (WES) data using an independent cohort of 108 prostate samples [17] (Pearson's $r=0.74$, $P<10$ e-15; Figure S5C in Additional file 7).

In terms of ploidy assessment we noted significant differences in the melanoma dataset where CLONET tends to undercall polyploidy (Figure S5D in Additional file 7). Where our conservative approach might definitely introduce false negative calls, we identified cases where close inspection of allele-specific data is not necessarily compatible with ABSOLUTE original calls of polyploidy [11]; for example, ME049T (Figure S5E in Additional file 7), where the relative distances among $\log \mathrm{R}$ peaks are more compatible with a diploid genome.

\section{Comparative analysis reveals different mechanisms of tumor deregulation}

We analyzed a total of 17,645 losses, 4,753 gains, 7,728 PMs, and 1,504 REARRs (Figure S6A in Additional file 8) and a panel of more than 23,000 genes from the RefSeq database [18]. Figure 5A summarizes the distribution of clonality across tumor types and aberrations. We compared the mean number of events classified as clonal or subclonal by means of the proportion test with BenjaminiHochberg false discovery rate (FDR) correction (Figure 5B; Table S2 in Additional file 5). We observed that deletions are more heterogeneous than gains in prostate and lung cancer (corrected $P$-values $<10 \mathrm{e}-6$ and $<10 \mathrm{e}-21$, respectively) and, interestingly, melanoma samples showed the opposite behavior (corrected $P$-value $<10 \mathrm{e}-21$ ). Moreover, when comparing the proportion of clonal/subclonal losses and gains across tumor types, the prostate and lung samples are statistically indistinguishable (corrected $P$-values of 0.49 and 0.365 , respectively). Overall, this suggests that temporally distinct mechanisms lead to loss and gain across the three tumor types. In terms of PMs, prostate cancer exhibits more subclonal events than melanoma, suggesting a more central role of PMs in melanoma oncogenesis compared with prostate cancer, as confirmed on an independent cohort from The Cancer Genome Atlas (TCGA) of 264 melanoma samples (Figure S6B in Additional file 8). However, aggregated values reflect only part of the story as great variability in the percentage of clonal events within a single combination of tumor and aberration is observed (Figure S6C in Additional file 8). We found no association between the patients' clinical 


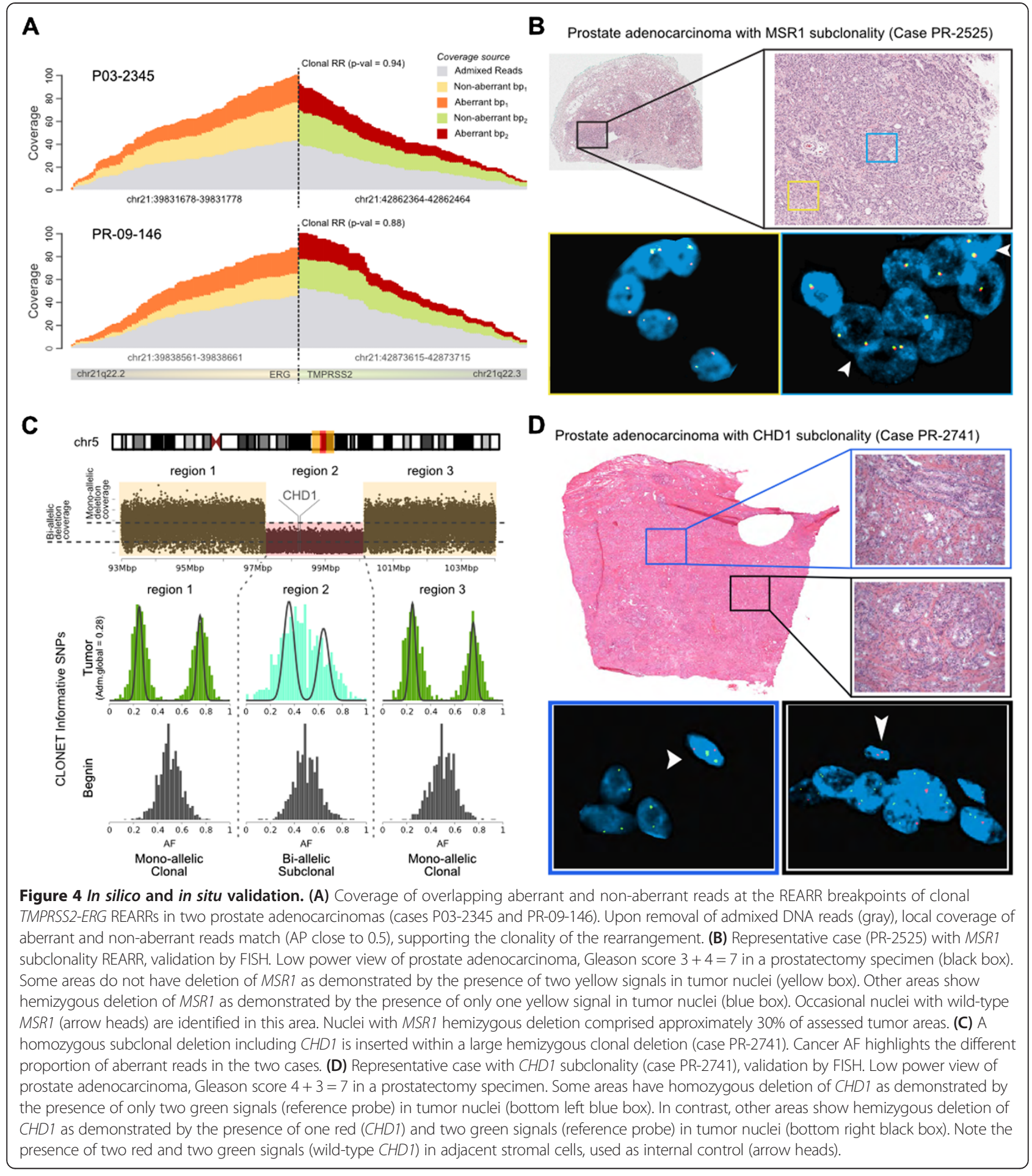

characteristics and exhibited clonal and subclonal range (Table S3 in Additional file 5).

Having assessed the variability in the clonality status of aberrations in individual patients, we then evaluated how it distributes along the whole genome as represented in clonality circos plots (Figure 6A). We can appreciate commonality among the three tumor types in some specific genomic regions. Genes on $8 \mathrm{p}$ are found clonally deleted in $96 \%, 100 \%$, and $100 \%$ of the prostate, melanoma, and lung samples, respectively. They include the prostate cancer suppressor NXK3-1 [19], the gene CSMD1, which is recurrently deleted in melanoma [11], and the phosphatase DUSP4, which is involved in negative feedback control of EGFR signaling in lung adenocarcinoma [20]. 

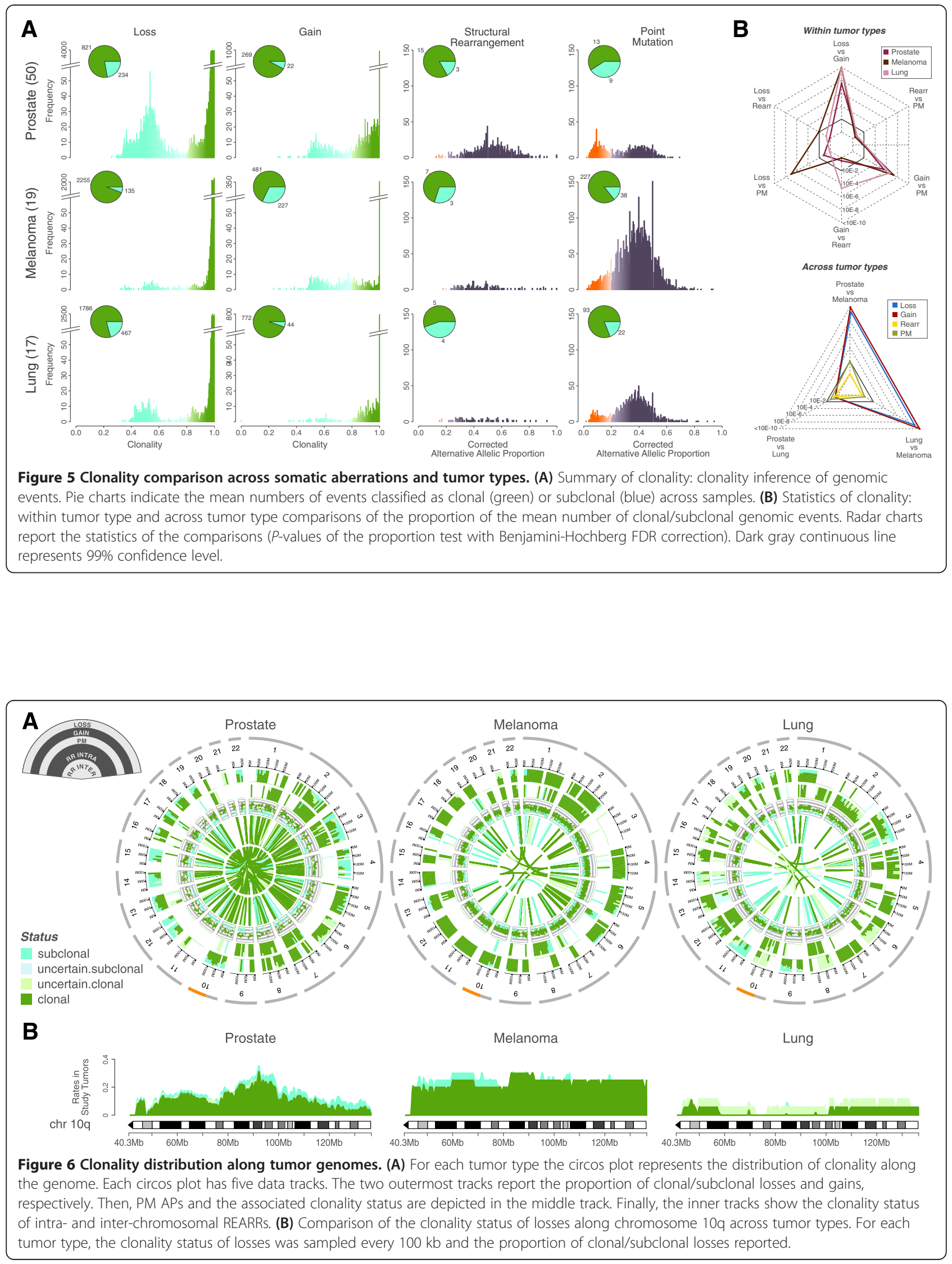
Then, we investigated whether clonality analysis can highlight tumor-specific mechanisms of deregulation. Our comparative analysis shows that although PTEN deletion is involved in many cancer types, the underlying timing of alteration may be different among tumor types (Figure 6B), and may point to differential roles for pathway inactivation. Specifically, the focal and subclonal deletion in the prostate samples suggests that evolutionary pressure is acting in this region later in the natural history of the disease and may promote cancer progression at a later stage of cancer evolution. In contrast, the broad and clonal deletion of a large part of chromosome 10q found in melanoma indicates that PTEN is homogenously lost in metastatic melanoma. In lung adenocarcinomas loss of PTEN expression in EGFR-mutant cells correlates with increased drug resistance [21], but the rarity of the deletion observed entails the hypothesis that loss of expression is not due to a genomic alteration of PTEN.

Finally, our tool can also identify tumor lineage-specific subclonality. MUC4, encoding a protein acting to upregulate cell cycle inhibitor p27 through ERBB2 phosphorylation [22] and mutated in melanoma [11], is subclonal in the majority of samples ( 7 out of $12,58 \%$ ) on a background mean subclonality rate of $14 \%$ (Figure 5A) (adjusted binomial test $P$-value $<0.05$ ); this is similar for MUC2 and PCMTD1. The SOX2 gene, which encodes a transcription factor relevant to lung development and has been identified as a lineage-survival oncogene in lung squamous cells carcinomas [23] and over-expressed in advanced tumors [24], is detected as a subclonal gain in one (case LUAD-AEIUF) of two amplified samples, with possible implications for prognosis and risk stratification.

\section{Clonal hierarchy of genomic aberrations}

We next analyzed the temporal evolution of driver aberrations $[25,26]$ to build evolution maps (Figure 7A) capitalizing on the information from multiple individuals' samples in the absence of multiregion samples from the same individual [27]. Given the study sample size and the mutation frequencies, we built drafts of evolution maps by implementing the following rules. An arrow from aberration $A_{1}$ to aberration $A_{2}$ is drawn if (i) $A_{1}$ and $A_{2}$ co-occur in at least two samples, (ii) $A_{1}$ preceded $A_{2}$ in at least one sample, and (iii) $A_{2}$ does not precede $A_{1}$ in the considered dataset (Figure 7A).

Extended analysis of the prostate tumors for which deletion evolution paths were provisionally characterized [10] confirmed the temporal relation between the deletion of PTEN and TMPRSS2-ERG rearrangements (both via deletions and insertions in patient P01-28) in a coherent analysis framework. In addition, the improved sensitivity of CLONET now identifies additional genes whose loss precedes the homozygous deletion of $C H D 1$, namely NKX3-1, FOXO1, and RB1 (Figure 7A). To find support for the observed relations, we further analyzed 203 independent localized prostate cancer samples that underwent WES, including the Barbieri et al. dataset [17] and the interim TCGA prostate cancer cohort. We verified the vast majority of the relations depicted in Figure 7A (exceptions are BRCA2 with FOXO1 and RB1). It is worth mentioning that no contradictory relations were detected. In addition, in a set of patients treated with brachytherapy [28], we verified through FISH analysis that PTEN subclonal deletion is preferentially observed in the setting of $E R G$ rearrangement in line with other recent studies [29].

By querying recently reported gene sets $[30,31]$ and high confidence driver genes [32], the melanoma sample analysis revealed that clonal gains over chromosome 8q, including the proto-oncogene $M Y C$, precede a missense mutation of $N F 1$, a negative regulator of RAS signaling [30], and that deletions on $6 \mathrm{q}$ spanning the pro-apoptotic factor BCLAF1 (Bcl2-associated factor 1) consistently precede missense mutations of NBPF10 (chromosome 1q21.1) and RANBP2 (chromosome 2q12.3).

Similarly, we considered 32 high confidence driver genes [32] in the lung adenocarcinoma set (Figure 7A). CLONET identified a path that stems from the clonal aberration of gene FBN2 [33], either via deletion or missense mutation. $F B N 2$ disruption precedes the subclonal deletion of genes along chromosome 9p, including significantly mutated known lung adenocarcinoma genes SMARCA4, KEAP1, and STK11 [12]. Interestingly, two paths involving different aberration mechanisms appear to lead to 9p deletion: (i) a direct path when the FBN2 gene is deleted; and (ii) an indirect one through the loss of the 3p21.31-22.1 genomic region in the case of a missense mutation of FBN2.

In order to investigate common patterns of progression across tumor types, we interrogated a large set of putative cancer genes $(\mathrm{N}=507$; September 2013 version of COS$\mathrm{MIC}$ cancer gene census [34]) and applied pairwise intersections (three supporting samples across the two tumor types) of identified paths (Additional file 9). Despite the conservative approach and the overall limited number of samples, we observed that $R B 1$ loss is consistently sequential to $6 \mathrm{q}$ losses in both melanomas and prostate cancers. Similarly, aberrations along 17q (TP53) are followed by NFKB2 loss, supporting additional oncogenic effects [35]. The same cluster of $17 \mathrm{q}$ gene aberrations precedes deletions along 12q24 in both prostate and lung samples. Last, we detected consistent subclonal deletions on $9 \mathrm{q}$ in both melanoma and lung samples following deletions on $15 q$ and IRF4 gain. Notably, the path included a subclonal deletion of the tumor suppressor TSC1 (tuberous sclerosis 1) that may activate the mammalian target of rapamycin (mTOR) pathway and promote tumor development [36].

Finally, we explored the evolution of known cancer signaling pathways (Figure 7B); both common themes across tumor types and tissue-specific patterns emerged. 


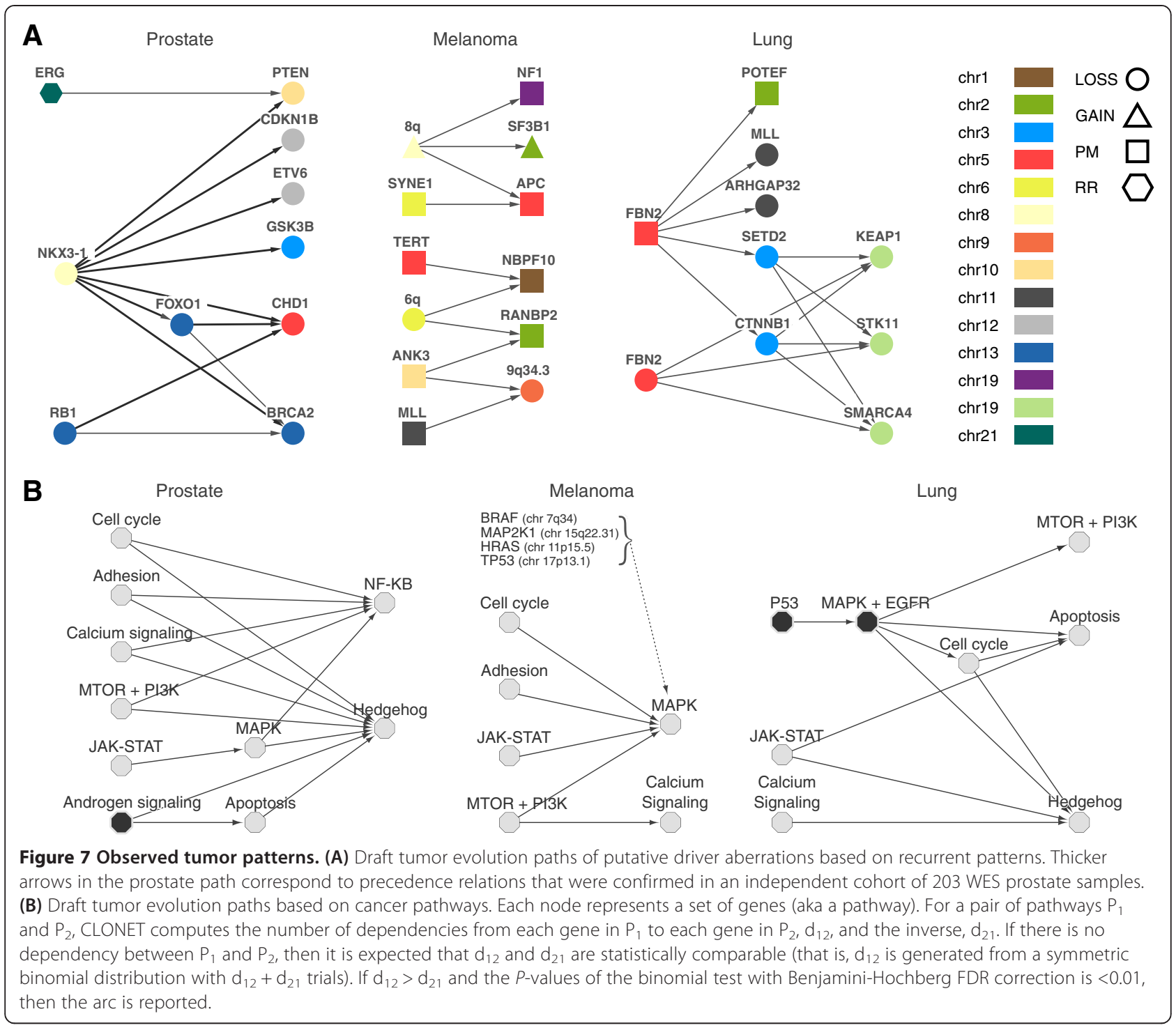

Well-established, recurrently deregulated pathways were detected as early drivers, such as androgen signaling in prostate cancer, core mitogen-activated protein kinase (MAPK) pathway components (such as BRAF) in melanoma, and $p 53$ and EGFR in lung cancer. Interestingly, although disruption of the same oncogenic pathways may be implicated across the three tumor types, the timing of dysregulation along the evolutionary paths can be independent.

\section{Discussion}

We have developed a mathematical model that exploits the genetic background of each individual to characterize cancer cell heterogeneity within a tumor specimen and builds lesion hierarchies by learning from recurrent patterns across multiple patients. The approach relies on base level information from a range of next-generation sequencing data (WGS, WES and targeted sequencing) and utilizes a local optimization approach and confidence propagation steps to enable the processing of complex subclonal patterns typical of patients with advanced tumors who may have undergone multiple treatment cycles.

The local optimization approach ensures accurate tumor purity assessment across challenging samples. Our group recently implemented CLONET in precision medicine WES reports to assess adequacy of biopsy samples, highly relevant in a regulatory compliant environment. Direct assessment of CLONET performance on WES versus WGS data from 15 samples (data from $[10,17])$ showed excellent agreement for Adm.global (Figure S8A in Additional file 10) and clonality estimates (Figure S8B in Additional file 10) where 97.6\% of genes annotated as subclonal in WES are also subclonal in WGS. This approach can also be utilized to assess 
temporal evolution from serial samples from one patient, as observed with patient 7520 , in which both his primary untreated prostate cancer and metastatic treatmentresistant tumor (biopsied three years later) were profiled. Upon correction for ploidy and global admixture, CLONET identified the gene AURKA as copy number neutral in the primary sample but found a gain of two copies in the late metastatic sample (Additional file 11). Amplification of AURKA has been described in treatmentresistant metastatic prostate cancer [29,37], and inhibitors of AURKA are currently in clinical trials. In addition, by exploiting the local optimization approach, we were successful in following tumor dynamics across cell-free DNA samples (liquid biopsies) from castration-resistant prostate cancer patients upon deep targeted sequencing and to monitor clonal expansion and contraction of key somatic aberration from low tumor content DNA samples [38].

Here, we report the results from three common solid tumor types characterized by diverse histology and clinical course and for which we were able to detect patterns and dynamics of subclonal evolution that support the relevance of the approach. Globally, we observed great variability across and within tumor types. Targeted analyses revealed both commonalities and clear differences (Figure 6A). Notably, we identified the partial loss of chromosome $8 \mathrm{p}$ as a common early event in the three tumors, while the focused analysis on the long arm of chromosome 10 suggested different mechanisms of PTEN deregulation and participation in tumorigenesis. Finally, specific examples of subclonality, such as MUC4 PMs in melanoma and SOX2 gains in lung, have been identified. Understanding patterns of tumor evolution can aid in the rational development of more effective and early therapeutic approaches to directly target clonal events that are driving tumorigenesis, especially as multiple potentially actionable alterations are often identified within one tumor even at diagnosis.

It is important to note that to properly construct comprehensive tumor evolution maps, thousands of genomes are required to reach adequate statistical power when considering the range of frequencies of co-occurring aberrations. As sequencing data for multiple tumor samples and tumor types becomes accessible to the community, maps will be drafted and completed over multiple iterations. In turn, this will soon allow assigning an evolution time stamp to each new clinically profiled sample based on where the tumor genome fits into the evolution maps. Establishing a 'timeline' of cancer progression is critical for biomarkers development in the precision medicine era, allowing clinicians to more accurately gauge prognosis by adding a molecular measure of progression to standard staging and grading systems, which do not associate with molecular heterogeneity of samples (Table S3 in Additional file 5). Such an approach may allow improved clinical decision-making in a variety of cancer types, guiding the choice of management strategies and level of aggressive therapy based on how far the tumor has progressed at the genomic level.

In summary, we present a robust method that exploits next-generation sequencing data to classify somatic lesions based on their clonality within the tumor cell population. The utility of CLONET is related to transitioning next-generation sequencing efforts from the static evaluation of untreated tumor samples to the clinical arena of precision medicine where patients will be followed along a continuum of treatment modalities and a targeted therapy regimen is based on the understanding of driver mutations.

\section{Conclusions}

Distinguishing gatekeeper or driver mutations from passenger mutations is a high priority for understanding disease progression. Knowledge of the chronology of molecular alterations can provide important insights into defining the most clinically relevant mutations that characterize important milestones in cancer. Genome sequencing of cancer samples taken during the course of precision medicine might demonstrate a wider range of genomic heterogeneity than previously observed in international genome sequencing studies. These clinical samples demonstrate more heterogeneity and admixture of both tumor and non-tumor components. To aid in unraveling the critical temporal evolution of somatic aberrations in challenging clinical tumors, we developed CLONET, a computation tool that requires only few clonal events to precisely estimate tumor purity and ploidy and then nominates the hierarchy of genomic aberrations. We demonstrate that CLONET can determine the clonality of different types of somatic aberrations, including SCNAs, PMs, and REARRs, using either WGS or WES datasets. We anticipate that with the emergence of larger genomic datasets, CLONET could help map out the evolution of molecular alterations.

\section{Materials and methods \\ CLONET pipeline}

A schematic view of the CLONET pipeline is shown in Figure S10A in Additional file 12. For this study input data were obtained as follows. Read counts of informative SNPs were extracted from BAM files using an in-house procedure, SCNAs were detected using SegSeq [39] from tumor and normal sequencing-based data, PM coordinates were as in original corresponding manuscripts, and REARRs were identified by means of dRanger and Breakpointer [14]. Finally, to avoid germline background effects, we filtered out genes (approximately 4,000) that intersect significant (size greater than $2 \mathrm{~kb}$ ) known germline copy number variants [40]. 


\section{Fluorescence in situ hybridization validation of CLONET}

To assess genomic deletion, disruptive translocations or polyploidy we used locus-specific dual-color FISH assays following a previously described approach [41,42]. To assess subclonality, at least 200 nuclei per area were evaluated using a fluorescence microscope (Olympus BX51; Olympus Optical, Tokyo, Japan). The probes used for FISH assays were: SPRY2, 5' RP11-51 N22 to 3' RP11-478 F4; MSR1, 5' RP11-6O24 to 3' RP11-794E24; ERG, 5' BAC RP11-372O17 to 3' BAC RP11-24A11; reference probe on 10q25, BAC RP11-431P18).

\section{CLONET on exome and targeted sequencing data}

The local approach implemented in CLONET enables the analysis of samples with few SCNAs provided that informative SNP read counts and Log $\mathrm{R}$ values are available. As with WGS data, individual specific informative SNPs can be identified from matched normal DNA samples. Appropriate Log $\mathrm{R}$ values for exome genomic segments or for each targeted area can be obtained with platform-specific strategies and provided to CLONET as input. Specifically, in the case of exome data, array-based segmented data or SCNA segments directly inferred from exome data with recent well-performing tools [43] can be utilized. CLONET combines segment input with exomederived read counts to estimate purity and ploidy and then nominate subclonal aberrations based on sequencing data. In the case of targeted sequencing data, copy number calls derived using custom control regions and very high coverage $(>1,000 \mathrm{X})$ [38] allowed for CLONET-based clonality estimation even in the case of low tumor content $(<10 \%)$.

\section{Expected distribution of the allelic fraction of a genomic segment}

Consider a genomic segment that spans a set of informative SNPs for the individual of interest. For any such SNP with coverage cov, the total number of reads $r$ supporting the reference base (reference reads) is the sum of the neutral reads $\left(r_{n}\right)$ and the active reads $\left(r_{a}\right)$ supporting the reference base. We define $\beta$ as the ratio between neutral reads and the total number of reads spanning the SNP of interest. The probability of having $k$ reference reads is then defined as the convolution of the probability of observing $\beta * k$ neutral reads and $(1-\beta)^{*} k$ active reads, that is:

$$
P(r=k, 0 \leq k \leq \operatorname{cov})=\operatorname{Conv}\left(P\left(r_{n}=\beta^{*} k\right), P\left(r_{a}=(1-\beta) * k\right)\right)
$$

We assume that $P\left(r_{n}=\beta * k\right)$ follows a binomial distribution with number of trials equal to $\beta * \mathrm{Cov}$ and probability of success equal to $p s$ (that is, the probability to observe a reference read). Note that $p s$ may deviate from 0.5 due to read-mapping biases [44]. All the active reads either support the reference base or the alternative base as only one allele is represented by definition of active reads. We define $N_{r e f}$ as the proportion of informative SNPs within the aberration that carry the SNP reference base in the allele represented by active reads (active allele). Then, the distribution $P\left(r_{a}=(1-\beta) * k\right)$ follows a categorical distribution with values equal to $N_{\text {ref }}$ if $r_{a}=(1-\beta) * k$ and equal to $\left(1-N_{r e f}\right)$ if $r_{a}=0$. Equation 2 can be written in a closed form as the sum of two binomial distributions (proof in Additional file 1):

$$
\begin{aligned}
P\left(r=k \mid \operatorname{cov}, \beta, N_{r e f}, p s\right)= & \left(1-N_{r e f}\right) * B\left(k \mid \beta^{*} \operatorname{cov}, p s\right) \\
& +N_{r e f} * B\left(k-(1-\beta) * \operatorname{cov} \mid \beta^{*} \operatorname{cov}, p s\right)
\end{aligned}
$$

where $B(m \mid n, p)$ is the probability mass function of a binomial distribution, that is, the probability of $m$ successes in $n$ trials with success probability $P$.

\section{Estimated proportion of neutral reads for a genomic segment}

The unknown values $\beta$ and $N_{\text {ref }}$ of Equation 3 can be inferred from the sequencing coverage at informative SNPs within the considered segment. In particular, given a segment Seg and a set $I$ of informative SNPs within Seg, each informative SNP in $I$ is a sample from the distribution of Equation 3. Optimization can be used to determine the values of $\beta$ and $N_{\text {ref }}$ for each segment. Given a random pair $\left(\beta, N_{r e f}\right)$, the Kolmogorov-Smirnov nonparametric goodness-of-fit test for discrete null distribution [45] computes the likelihood that the informative SNPs in $I$ are a sample from $P\left(r=k \mid c, \beta, N_{r e f}, p s\right)$. Next, a particle swarm optimization method [13] finds a candidate pair $\hat{\beta}, \hat{N}_{\text {ref }}$ that best represents the distribution of the allelic fraction of the SNPs in $I$.

\section{From neutral reads to non-aberrant reads}

Consider a genomic segment $\mathrm{Seg}$. If the $\mathrm{Log} \mathrm{R}$ value of $\mathrm{Seg}$ supports a SCNA C, we define as aberrant those reads that cover Seg and are sequenced from cells harboring $\mathrm{C}$. If $\mathrm{Seg}$ is a candidate somatic mono-allelic deletion, the percentage of neutral reads $\beta$ corresponds to the percentage of reads that cover $\mathrm{Seg}$ and are sequenced from cells harboring both alleles, that is, neutral and non-aberrant reads correspond. If the Log R value of Seg supports a gain with integer copy number $c n>2$, we have to re-scale $\beta$ to obtain the percentage of sequenced cells that have copy number $c n$ (that is, the percentage of non-aberrant reads). For the sake of simplicity, we reason in terms of at most one copy difference between alleles. If $c n$ is odd, the number of neutral reads is the sum of the neutral reads from admixed cells plus the neutral reads of the gain (Figure S10B in Additional file 12). The percentage $\beta_{c n}$ of reads from cells with copy number $c n$ is computed 
from the percentage of neutral reads $\beta$ by removing neutral reads due to the gain, that is:

$$
\beta_{c n}=1-c n_{G} *(1-\beta)
$$

If $c n$ is even and at most one copy difference between alleles is allowed, then $\beta$ is close to one, as both alleles are equally represented. This reasoning applies to any arbitrary combinations of the number of alleles (Figure S10C in Additional file 12).

\section{From aberrant reads to aberrant cells}

Given a somatic mono-allelic deletion $M$, the local admixture Adm.local is defined as the proportion of cells not harboring $M$ (non-aberrant cells) over the total number of cells. Let $a$ define the total number of reads supporting the alternative allele (alternative reads), as the sum of neutral reads $\left(a_{n}\right)$ and active reads $\left(a_{a}\right)$ supporting the alternative base. For any informative SNP within $M$, the local admixture at $M$ results:

$$
\text { Adm.local }{ }_{M}=\frac{\frac{r_{n}+a_{n}}{2}}{\frac{r_{n}+a_{n}}{2}+\left(r_{a}+a_{a}\right)}
$$

and the proportion of non-aberrant reads covering $M$ is:

$$
\beta_{M}=\frac{r_{n}+a_{n}}{r_{n}+a_{n}+r_{a}+a_{a}}
$$

By combining Equations 5 and 6, we can prove Equation 1. Additional file 1 reports a full proof of a generalized version of Equation 1 that accounts for any positive copy number.

\section{Uncertainty assessment and its propagation to clonality estimates}

Different error sources introduce a bias into the distribution of the allelic fraction that may lead to inaccurate estimates of $\beta$. To optimize sensitivity and specificity, we compute the estimation uncertainty $\varepsilon$ around $\beta$ and we provide a sound way to propagate $\varepsilon$ to clonality values. The value of $\varepsilon$ varies based upon the depth of sequence mean coverage (mean.cov) and the number of available informative SNPs (n.info.snp) across the segment of interest in the sample considered. The mean.cov controls the ability to discern the two modes of the AF distribution: the inset of Figure 2A shows that the higher $\beta$ is, the more coverage is needed. We evaluated the uncertainty in $\beta$ estimates as a function of these parameters by randomly generating 50 samples with given mean.cov, n.info.snps, and $\beta$ and averaging the difference between the expected and computed $\beta$ for a given coverage and number of SNPs. Accordingly, we generated a look-up uncertainty table for each combination of mean.cov and n.info.snps (Figure 3A).
The procedure to infer the value of $\beta$ of a segment is independent of its $\log \mathrm{R}$ value. Interestingly, if we plot the $\log \mathrm{R}$ of a segment versus its value of $\beta$, segments aggregate into clusters, where each cluster corresponds to a specific copy number and to a definite clonality status (Figure S10C in Additional file 12). If we restrict our attention to putative somatic mono-allelic deletions, the cluster $B_{\min }$ with the lowest median value of $\beta$ would likely represent $100 \%$ clonal deletions. CLONET relies on an uncertainty table (Figure $3 \mathrm{~A}$ ) to characterize $B_{\text {min }}$. We define $B$ as the set of $\beta$ values of all the putative somatic mono-allelic deletions and $\operatorname{err}(\beta)$ as the uncertainty around $\beta$. In this context, $B_{\min }$ is defined as the smallest subset of $B$ such that $\min (B)$ in $B_{\min }$ and for all $\beta^{\prime}$ in $B$ and not in $B_{\text {min }}, \max \left(B_{\text {min }}\right)+\operatorname{err}\left(\max \left(B_{\text {min }}\right)\right)<\beta^{\prime}-$ $\operatorname{err}\left(\beta^{\prime}\right)$. CLONET starts from $\min (B)$ and searches for a $\beta$ ' value such that their difference is not explained by the error table. Then, CLONET selects the median value of $B_{\text {min }}$ as candidate Adm.global and computes the uncertainty around Adm.global as the minimum and the maximum of the $\lambda$ percentage of the distribution of $B_{\text {min }}$. The value of $\lambda$ depends on the noise observed in the coverage (in this study we set $\lambda$ equal to $95 \%$ ).

Given a somatic copy number $C$ in a tumor sample, CLONET computes its local (Adm.local $C$ ) and global (Adm.global) admixture. The clonality $\mathrm{Cl}_{C}$ of $C$ is then defined as the percentage of tumor cells in a sample harboring $C$ :

$$
C l_{C}=\frac{1-\text { Adm.local }_{C}}{1-A d m \cdot \text { global }}
$$

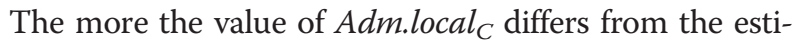
mated global admixture Adm.global, the more $C$ is subclonal, namely the value of $\mathrm{Cl}_{C}$ approximates to 0 . Finally, interval analysis [46] allows propagating uncertainty around $\beta$ and Adm.global to clonality (Figure S10D in Additional file 12).

\section{Clonality of bi-allelic deletions}

Equation 7 enables us to compute the percentage of tumor cells harboring any specific aberration (that is, its clonality) except for clonal bi-allelic deletions. For subclonal bi-allelic deletions the allelic fraction signal comes from cells with either two or one allele (Additional file 2). Consider a subclonal bi-allelic deletion where $n$ (normal), $m$ (mono-allelic), and $b$ (bi-allelic) denote the proportion of cells with two, one, and zero alleles, respectively $(n+m+$ $b=1)$. By Equation 1 one can assess a local estimate of the admixture (Adm.local) that represents the proportion of cells with two alleles in the subpopulation of cells with one or two alleles, that is $n=\operatorname{Adm}_{\text {local }} *(n+m)$. We can also observe that the proportion of normal cells $n$ in the sample is equal to the global DNA admixture Adm.global. 
Finally, we define the clonality of a bi-allelic deletion $C l_{B}$ as the percentage of cells harboring a bi-allelic deletion over the number of cells with a mono- or a bi-allelic deletion, that is, $b /(m+b)$. One can prove (Additional file 1 ) that the clonality $\mathrm{Cl}_{b}$ of a bi-allelic deletion is:

$$
C l_{b}=\frac{\text { Adm.global }- \text { Adm.local } \times \text { Adm.global }}{\text { Adm.local } \times(1-\text { Adm.global })}
$$

\section{Data availability}

Binary sequence alignment/map (BAM) files of WGS data are accessible in the database of Genotypes and Phenotypes (dbGaP) with accession numbers phs000447. v1.p1 (prostate cancer [10]), phs000452.v1.p1 (melanoma [11]), and phs000488.v1.p1 (lung [12]); BAMs of WES data at dbGaP with accession number phs000447.v1.p1 (prostate cancer [17]).

\section{CLONET source code}

Source code is available at [47] and at the versioncontrolled repository [48].

\section{Additional files}

\section{Additional file 1: Supplementary text.}

Additional file 2: Figure S1. Pictorial representation of the method CLONET uses to manage bi-allelic deletions. Three types of cells are considered: normal cells (yellow) with gene A (dark brown) and gene B (light brown) present in two copies; tumor cells of type I (light red) harbor a bi-allelic deletion of both genes A and B; tumor cells of type II (dark red) have zero copies of $B$ and one copy of $A$. The bottom row reports the distribution of the expected AF at informative SNPs within gene $A$ and gene B. In pure diploid cells with two copies of genes $A$ and B, AF is centered at 0.5. In type I tumor cells, there is no signal, as both alleles are deleted. In type II tumor cells, one allele of gene B is present and the AF assumes values 0 or 1 . In a hypothetical mixture of normal and tumor cells (right panel), the distribution of AFs along gene A reports only the signal from the DNA admixture, while the distribution of gene B corresponds to a mono-allelic deletion, reflecting the fact that cells with a bi-allelic deletion do not contribute to the AF.

Additional file 3: Figure S2. (A-C) Histograms of the Log R data of all the samples in the prostate (A), melanoma (B), and lung datasets $(C)$. The left plot shows data as reported by the segmentation algorithm while the right plot shows Log $\mathrm{R}$ values after ploidy and Adm.global correction. Log $R$ correction improves the quality of the segmentation and simplify the detection of copy number aberrations.

Additional file 4: Figure S3. (A) Comparison between alternative allele proportions computed from WGS and MiSeq experiments. Scatterplot of the alternative allelic proportion (AP) on 18 somatic point mutations in prostate samples selected for MiSeq validation. The $x$-axis reports the AP observed on MiSeq data and the $y$-axis reports the same value computed on WGS data (Table S1 in Additional file 5). The color of a point corresponds to the clonality assigned by CLONET to the point mutation. Inset text reports Pearson product-moment correlation coefficient and associated $P$-value. (B) Allelic fraction (AF) of informative SNPs along the interstitial deletion between TMPRSS2 and ERG and of an independent control clonal deletion for each sample reported in Figure 3A. The clonality statuses of the REARRs and of the accompanying interstitial deletions are identical.

Additional file 5: Table S1. Read count of selected point mutations for MiSeq validation. Table S2. Pairwise comparison of the percentage of subclonal genomic events relative to Figure 5C. Table S3. The association between the percentage of subclonal genomic events when samples are partitioned according to patient clinical characteristics. The table shows that the clinical characteristics are not able to distinguish between less and more heterogeneous samples.

Additional file 6: Figure S4. Experimental in situ validation. (A) Low power view of adenocarcinoma Gleason score $3+3=6$ in a prostatectomy specimen representative case of prostate adenocarcinoma with SPRY2 subclonality (case STID-3042). Some areas do not have deletion of $S P R Y 2$, as demonstrated by the presence of two yellow signals in tumor cells by FISH (yellow box). In contrast, other areas show hemizygous deletion of SPRY2, as demonstrated by the presence of only one yellow signal (blue box; arrow heads) in tumor cells by FISH. (B) Low power view of prostate adenocarcinoma Gleason score $4+4=8$ with tertiary Gleason pattern 5 in a prostatectomy specimen from a representative case of prostate adenocarcinoma with CHD1 subclonality (case STID 2525). Some areas have homozygous deletion of $\mathrm{CHD1}$ as demonstrated by the presence of only two yellow signals (reference probe) in tumor cells by FISH (yellow box). In contrast, other areas show hemizygous deletion of CHD1 as demonstrated by the presence of one red (CHD1) and two yellow signals (reference probe) in tumor cells by FISH (blue box). Note the presence of two red and two yellow signals (normal) in adjacent stromal cells, used as internal control (arrow heads).

Additional file 7: Figure S5. In silico validation. (A) Scatterplot of the Adm.global estimates of CLONET (y-axis) versus those of ABSOLUTE (x-axis). Each dot represents a WGS melanoma sample whose color corresponds to the ploidy value estimated by ABSOLUTE. The plot shows that the ploidy of a sample does not bias the estimation. Inset text reports Pearson product-moment correlation coefficient and associated P-value. (B) Scatterplot of the Adm.global estimates of CLONET (y-axis) versus those of ABSOLUTE ( $x$-axis) where each dot represents a WGS prostate sample. Inset text reports Pearson product-moment correlation coefficient and associated P-value. (C) Scatterplot of the Adm.global estimates of CLONET ( $y$-axis) versus those of ABSOLUTE ( $x$-axis) where each dot represents a WES prostate sample. Inset text reports Pearson product-moment correlation coefficient and associated $P$-value. Ploidy evaluation on the same dataset gives concordant values and found only an aneuploidy sample (case 04-1243 L). (D) Scatterplot of the ploidy estimates of CLONET (y-axis) versus those of ABSOLUTE (x-axis). Each dot represents a WGS melanoma sample whose color corresponds to the Adm.global value estimated by ABSOLUTE. The plot shows that the Adm. global of a sample does not bias the estimation. Inset text reports Pearson product-moment correlation coefficient and associated P-value. (E) A melanoma case (ME049T) classified as having ploidy equal to 3.05 by ABSOLUTE and equal to 1.93 by CLONET. The histogram (top) shows the Log R distribution of the segments. Yellow, violet, and orange arrows point to key Log $R$ peaks used by both CLONET and ABSOLUTE for ploidy estimation. Beta versus Log R plot (bottom) shows the observed values for each genomic segment in sample ME049T (gray dots) and the expected position given purity and ploidy estimated by CLONET and ABSOLUTE (blue and green dots, respectively). Boxes show allele specific copy number values defined by the position in the Beta versus Log $R$ space.

Additional file 8: Figure S6. (A) Summary of aberrations: genomic events (GE) characterized in three tumor datasets generated through whole genome sequencing. (B) Histogram of the alternative allelic proportion after Adm.global correction of the copy number neutral somatic point mutations detected in a cohort of 264 melanoma samples from TCGA. Pie chart indicates the mean numbers of events classified as clonal (green) or subclonal (blue) across samples. (C) Boxplot of the percentage of clonal genes across GEs and tumor types with respect to the total number of aberrant genes. Superimposed strip-charts represent per sample data: the size of each dot is proportional to the number of genes analyzed.

Additional file 9: Figure S7. Common evolution of cancer gene aberrations across tumor samples. Pairwise intersection of the tumor evolution paths of prostate, melanoma and lung samples computed on a panel of 507 cancer genes. Nodes stand for aberrant genes with the color representing the chromosome and the shape the kind of 
aberration. Arcs model temporal order between two aberrations found in at least three samples of the two tumor types considered. The central semicircle reports the dependencies found in the three tumor types.

Additional file 10: Figure S8. Comparison of WGS- and WES-based estimates. (A) Scatterplot of the Adm.global estimates of CLONET on 15 prostate patients for which both exome data (y-axis) and WGS data ( $\mathrm{x}$-axis) are available. Inset text reports Pearson product-moment correlation coefficient and associated $P$-value. (B) Scatterplot of the percentage of clonality estimated for 23,484 genes in 15 prostate samples computed using exome data (y-axis) and WGS data (x-axis). Inset text reports Pearson product-moment correlation coefficient and associated $P$-value.

Additional file 11: Figure S9. Case of tumor progression. (A,B) The top part of the figure shows histograms of the Log $R$ data for a primary prostate sample (A) and a pelvic mass metastasis (B) from the same patient. (C,D) Upon correction for ploidy and global admixture, CLONET identifies gene AURKA as copy number neutral in the primary sample (C) but found a gain of two copies in the late metastatic sample (D). (E) The shift in the Log R values prior and after CLONET ploidy correction in the metastatic sample indicates an aneuploidy genome, as confirmed by FISH analysis that demonstrate four yellow signals (reference probe) in tumor cells. The probes that were used for FISH assays are as follows: red test probe, 3' ERG (BAC RP11-24A11); reference probe, 10q25

(BAC RP11-431P18).

Additional file 12: Figure S10. CLONET method. (A) Schematic overview of the computing steps that lead to the definition of the tumor evolution path. (B) An example of a tumor specimen with two non-aberrant cells (yellow) and three aberrant cells (blue) with a duplicated genomic region (red). The Adm.global of this specimen is $2 / 5$ and the percentage of aberrant reads is $4 / 13$. Note that these values respect Equation 1. The left shows tumor cells that result from decomposing the blue aberrant cells into three normal cells and three aberrant cells with a mono-allelic deletion (brown). The percentage of neutral reads is 10/13. The value of $\beta$ is rescaled to account for the gain by considering the proportion of aberrant reads is three times greater, that is, $1-\left(3^{*}(1-\beta)\right)$. The bottom plot highlights that the AF of the tumor specimen and of its decomposition are the same. (C) Example of the distribution of the expected $\beta$ versus Log $R$ values in a sample with $20 \%$ of Adm.global and a mean ploidy of 2. Each point represents a genomic segment defined by its Log $R$ value, computed by segmentation, and its $\beta$ value, computed by CLONET. In particular, the blue cluster includes segments where only one allele is present in $100 \%$ of the tumor cell population (that is, they are mono-allelic clonal deletions). These segments are used to compute the Adm.global of the sample. The variability range of the Adm.global returned by CLONET considers the dispersion of the data in this cluster. (D) The plot shows how the variability ranges of local and global DNA admixture estimates propagate to the clonality values. Each box corresponds to a pair of local and global DNA admixture values and illustrates the clonality variability range as a function of their variability ranges. Local and global admixture variability ranges are computed from the $\beta$ uncertainty table.

\section{Abbreviations}

AF: allelic fraction (fraction of reads supporting the reference base); AP: proportion of reads supporting the alternative allele or alternative allelic proportion (fraction of reads supporting the alternative allele at a somatic point mutation position); BAF: B allelic fraction (refer to array based data); CLONET: CLONality Estimate in Tumors; FDR: false discovery rate; FISH: fluoresce in situ hybridization; PM: somatic point mutation; REARR: structural rearrangement; SCNA: somatic copy number aberration; SNP: single-nucleotide polymorphism; TCGA: The Cancer Genome Atlas; WES: whole exome sequencing; WGS: whole genome sequencing.

\section{Competing interests}

The authors declare that they have no financial competing interests.

\section{Authors' contributions}

FD and DP conceived the local optimization approach to handle genomic heterogeneity and designed the study analysis. DP designed and implemented CLONET, and performed data analysis. SB contributed to the design of REARRs analysis and performed MiSeq validation experiments. AR assisted with informative SNP identification and implemented REARR relevant read detection. CEB and $\mathrm{HB}$ assisted with temporal evolution and pathway-based analysis. JMM and JF performed in situ validation experiments. $C E B, A S, L A G$, and MAR contributed to the interpretation of the results and contributed to the final manuscript. DP, MAR and FD wrote the manuscript and FD provided leadership for the project. All authors read and approved the final manuscript.

\section{Acknowledgements}

We like to thank TY MacDonald for technical support, E Van Allen for fruitful discussions, and $\mathrm{O}$ Elemento for critical reading of the manuscript.

\section{Financial support}

This work was supported by the following grants: US Department of Defense Synergy Awards (PC101020 to FD, LAG, and MAR), US National Cancer Institute (2R01CA125612-05A1 to MAR and FD), the Prostate Cancer Foundation, the Prostate Cancer Foundation Young Investigator Award (CEB), Early Detection Research Network US NCI CA111275 (MAR, JMM). Tissue-based work was supported in part by the Translational Research Program at WCMC, Department of Pathology and Laboratory Medicine.

\section{Author details}

${ }^{1}$ Centre for Integrative Biology, University of Trento, Povo Trento 38123, Italy. ${ }^{2}$ The Broad Institute of MIT and Harvard, Cambridge, MA 02141, USA. ${ }^{3}$ Dana-Farber Cancer Institute, Boston, MA 02215, USA. ${ }^{4}$ Harvard Medical School, Boston, MA 02115, USA. ${ }^{5}$ Department of Urology, Weill Medical College of Cornell University, New York, NY 10065, USA. ${ }^{6}$ Department of Pathology and Laboratory Medicine, Weill Medical College of Cornell University, New York, NY 10065, USA. ${ }^{7}$ Department of Medicine, Division of Hematology and Oncology, Weill Medical College of Cornell University, New York, NY 10065, USA. ${ }^{8}$ Institute for Precision Medicine, Weill Medical College of Cornell University and New York Presbyterian Hospital, New York, NY 10065, USA. ${ }^{9}$ HRH Prince Alwaleed Bin Talal Bin Abdulaziz Alsaud Institute for Computational Biomedicine, Weill Medical College of Cornell University, New York, NY 10065, USA.

Received: 14 July 2014 Accepted: 13 August 2014 Published online: 26 August 2014

\section{References}

1. Greenman CD, Bignell G, Butler A, Edkins S, Hinton J, Beare D, Swamy S, Santarius T, Chen L, Widaa S, Futreal PA, Stratton MR: PICNIC: an algorithm to predict absolute allelic copy number variation with microarray cancer data. Biostatistics 2010, 11:164-175.

2. Li A, Liu Z, Lezon-Geyda K, Sarkar S, Lannin D, Schulz V, Krop I, Winer E, Harris L, Tuck D: GPHMM: an integrated hidden Markov model for identification of copy number alteration and loss of heterozygosity in complex tumor samples using whole genome SNP arrays. Nucleic Acids Res 2011, 39:4928-4941.

3. Van Loo P, Nordgard SH, Lingjaerde OC, Russnes HG, Rye IH, Sun W, Weigman VJ, Marynen P, Zetterberg A, Naume B, Perou CM, Børresen-Dale A-L, Kristensen VN: Allele-specific copy number analysis of tumors. Proc Natl Acad Sci U S A 2010, 107:16910-16915.

4. Yau C, Mouradov D, Jorissen RN, Colella S, Mirza G, Steers G, Harris A, Ragoussis J, Sieber O, Holmes CC: A statistical approach for detecting genomic aberrations in heterogeneous tumor samples from single nucleotide polymorphism genotyping data. Genome Biol 2010, 11:R92.

5. Carter SL, Cibulskis K, Helman E, McKenna A, Shen H, Zack T, Laird PW, Onofrio RC, Winckler W, Weir BA, Beroukhim R, Pellman D, Levine DA, Lander ES, Meyerson M, Getz G: Absolute quantification of somatic DNA alterations in human cancer. Nat Biotechnol 2012, 30:413-421.

6. Nik-Zainal S, Van Loo P, Wedge DC, Alexandrov LB, Greenman CD, Lau KW, Raine K, Jones D, Marshall J, Ramakrishna M, Shlien A, Cooke SL, Hinton J, Menzies A, Stebbings LA, Leroy C, Jia M, Rance R, Mudie LJ, Gamble SJ, Stephens PJ, McLaren S, Tarpey PS, Papaemmanuil E, Davies HR, Varela I, McBride DJ, Bignell GR, Leung K, Butler AP, et al: The life history of 21 breast cancers. Cell 2012, 149:994-1007.

7. Oesper L, Mahmoody A, Raphael BJ: THetA: inferring intra-tumor heterogeneity from high-throughput DNA sequencing data. Genome Biol 2013, 14:R80. 
8. Strino F, Parisi F, Micsinai M, Kluger Y: TrAp: a tree approach for fingerprinting subclonal tumor composition. Nucleic Acids Res 2013, 41:e165.

9. Yau C: OncoSNP-SEQ: a statistical approach for the identification of somatic copy number alterations from next-generation sequencing of cancer genomes. Bioinformatics 2013, 29:2482-2484.

10. Baca SC, Prandi D, Lawrence MS, Mosquera JM, Romanel A, Drier Y, Park K, Kitabayashi N, MacDonald TY, Ghandi M, Van Allen E, Kryukov GV, Sboner A, Theurillat J-P, Soong TD, Nickerson E, Auclair D, Tewari A, Beltran H, Onofrio RC, Boysen G, Guiducci C, Barbieri CE, Cibulskis K, Sivachenko A, Carter SL, Saksena G, Voet D, Ramos AH, Winckler W, et al: Punctuated evolution of prostate cancer genomes. Cell 2013, 153:666-677.

11. Berger MF, Hodis E, Heffernan TP, Deribe $Y L$, Lawrence MS, Protopopov A, Ivanova E, Watson IR, Nickerson E, Ghosh P, Zhang H, Zeid R, Ren X, Cibulskis K, Sivachenko AY, Wagle N, Sucker A, Sougnez C, Onofrio R, Ambrogio L, Auclair D, Fennell T, Carter SL, Drier Y, Stojanov P, Singer MA, Voet $D$, Jing R, Saksena G, Barretina J, et al: Melanoma genome sequencing reveals frequent PREX2 mutations. Nature 2012, 485:502-506.

12. Imielinski $M$, Berger $A H$, Hammerman PS, Hernandez B, Pugh TJ, Hodis $E$, Cho J, Suh J, Capelletti M, Sivachenko A, Sougnez C, Auclair D, Lawrence MS, Stojanov P, Cibulskis K, Choi K, de Waal L, Sharifnia T, Brooks A, Greulich $H$, Banerji S, Zander T, Seidel D, Leenders F, Ansén S, Ludwig C, Engel-Riedel W, Stoelben E, Wolf J, Goparju C, et al: Mapping the hallmarks of lung adenocarcinoma with massively parallel sequencing. Cell 2012, 150:1107-1120.

13. Kennedy J: Particle swarm optimization. In Encyclopedia of Machine Learning. Edited by Sammut C, Webb G. US: Springer; 2010:760-766.

14. Drier Y, Lawrence MS, Carter SL, Stewart C, Gabriel SB, Lander ES, Meyerson M, Beroukhim R, Getz G: Somatic rearrangements across cancer reveal classes of samples with distinct patterns of DNA breakage and rearrangement-induced hypermutability. Genome Res 2013, 23:228-235.

15. Tomlins SA, Rhodes DR, Perner S, Dhanasekaran SM, Mehra R, Sun XW Varambally S, Cao X, Tchinda J, Kuefer R, Lee C, Montie JE, Shah RB, Pienta $\mathrm{KJ}$, Rubin MA, Chinnaiyan AM: Recurrent fusion of TMPRSS2 and ETS transcription factor genes in prostate cancer. Science 2005, 310:644-648.

16. Perner S, Demichelis F, Beroukhim R, Schmidt FH, Mosquera JM, Setlur S, Tchinda J, Tomlins SA, Hofer MD, Pienta KG, Kuefer R, Vessella R, Sun X-W, Meyerson M, Lee C, Sellers WR, Chinnaiyan AM, Rubin MA: TMPRSS2:ERG fusion-associated deletions provide insight into the heterogeneity of prostate cancer. Cancer Res 2006, 66:8337-8341.

17. Barbieri CE, Baca SC, Lawrence MS, Demichelis F, Blattner M, Theurillat JP, White TA, Stojanov P, Van Allen E, Stransky N, Nickerson E, Chae S-S, Boysen G, Auclair D, Onofrio RC, Park K, Kitabayashi N, MacDonald TY, Sheikh K, Vuong T, Guiducci C, Cibulskis K, Sivachenko A, Carter SL, Saksena G, Voet D, Hussain WM, Ramos AH, Winckler W, Redman MC, et al: Exome sequencing identifies recurrent SPOP, FOXA1 and MED12 mutations in prostate cancer. Nat Genet 2012, 44:685-689.

18. Pruitt KD, Tatusova T, Brown GR, Maglott DR: NCBI Reference Sequences (RefSeq): current status, new features and genome annotation policy. Nucleic Acids Res 2012, 40:D130-D135.

19. Bhatia-Gaur R, Donjacour AA, Sciavolino PJ, Kim M, Desai N, Young P, Norton CR, Gridley T, Cardiff RD, Cunha GR, Abate-Shen C, Shen MM: Roles for Nkx3.1 in prostate development and cancer. Genes Dev 1999, 13:966-977.

20. Chitale D, Gong Y, Taylor BS, Broderick S, Brennan C, Somwar R, Golas B, Wang L, Motoi N, Szoke J, Reinersman JM, Major J, Sander C, Seshan VE, Zakowski MF, Rusch V, Pao W, Gerald W, Ladanyi M: An integrated genomic analysis of lung cancer reveals loss of DUSP4 in EGFR-mutant tumors. Oncogene 2009, 28:2773-2783.

21. Paolini JB, Mancini J, Genestal M, Gonzalez H, McKay RE, Samii K, Fourcade OA: Predictive value of abdominal obesity vs. body mass index for determining risk of intensive care unit mortality. Crit Care Med 2010, 38:1308-1314.

22. Jepson S, Komatsu M, Haq B, Arango ME, Huang D, Carraway CA, Carraway $\mathrm{KL}$ : Muc4/sialomucin complex, the intramembrane ErbB2 ligand, induces specific phosphorylation of ErbB2 and enhances expression of p27(kip), but does not activate mitogen-activated kinase or protein kinaseB/Akt pathways. Oncogene 2002, 21:7524-7532.

23. Bass AJ, Watanabe $H$, Mermel CH, Yu S, Perner S, Verhaak RG, Kim SY, Wardwell L, Tamayo P, Gat-Viks I, Ramos AH, Woo MS, Weir BA, Getz G, Beroukhim R, O'Kelly M, Dutt A, Rozenblatt-Rosen O, Dziunycz P, Komisarof J, Chirieac LR, Lafargue CJ, Scheble V, Wilbertz T, Ma C, Rao S, Nakagawa H, Stairs DB,
Lin L, Giordano TJ, et al: SOX2 is an amplified lineage-survival oncogene in lung and esophageal squamous cell carcinomas. Nat Genet 2009, 41:1238-1242.

24. Sholl LM, Barletta JA, Yeap BY, Chirieac LR, Hornick JL: Sox2 protein expression is an independent poor prognostic indicator in stage I lung adenocarcinoma. Am J Surg Pathol 2010, 34:1193-1198.

25. Davoli T, Xu AW, Mengwasser KE, Sack LM, Yoon JC, Park PJ, Elledge SJ: Cumulative haploinsufficiency and triplosensitivity drive aneuploidy patterns and shape the cancer genome. Cell 2013, 155:948-962.

26. Gonzalez-Perez A, Perez-Llamas C, Deu-Pons J, Tamborero D, Schroeder MP, Jene-Sanz A, Santos A, Lopez-Bigas N: IntOGen-mutations identifies cancer drivers across tumor types. Nat Methods 2013, 10:1081-1082.

27. Gerlinger M, Rowan AJ, Horswell S, Larkin J, Endesfelder D, Gronroos E, Martinez P, Matthews N, Stewart A, Tarpey P, Varela I, Phillimore B, Begum S, McDonald NQ, Butler A, Jones D, Raine K, Latimer C, Santos CR, Nohadani M, Eklund AC, Spencer-Dene B, Clark G, Pickering L, Stamp G, Gore M, Szallasi Z, Downward J, Futreal PA, Swanton C: Intratumor heterogeneity and branched evolution revealed by multiregion sequencing. N Engl J Med 2012, 366:883-892.

28. Fontugne J, Lee D, Cantaloni C, Barbieri CE, Caffo O, Hanspeter E, Mazzoleni G, Dalla Palma P, Rubin MA, Fellin G, Mosquera JM, Barbareschi M, Demichelis F: Recurrent prostate cancer genomic alterations predict response to brachytherapy treatment. Cancer Epidemio/ Biomarkers Prev 2014, 23:594-600.

29. Krohn A, Freudenthaler F, Harasimowicz S, Kluth M, Fuchs S, Burkhardt L, Stahl P, CT M, Bauer M, Tennstedt P, Graefen M, Steurer S, Sirma H, Sauter G, Schlomm T, Simon R, Minner S: Heterogeneity and chronology of PTEN deletion and ERG fusion in prostate cancer. Mod Pathol 2014, doi:10.1038/modpathol.2014.70.

30. Hodis E, Watson IR, Kryukov GV, Arold ST, Imielinski M, Theurillat JP, Nickerson E, Auclair D, Li L, Place C, Dicara D, Ramos AH, Lawrence MS Cibulskis K, Sivachenko A, Voet D, Saksena G, Stransky N, Onofrio RC, Winckler W, Ardlie K, Wagle N, Wargo J, Chong K, Morton DL, Stemke-Hale K, Chen G, Noble M, Meyerson M, Ladbury JE, et al: A landscape of driver mutations in melanoma. Cell 2012, 150:251-263.

31. Lawrence MS, Stojanov P, Mermel CH, Robinson JT, Garraway LA, Golub TR, Meyerson M, Gabriel SB, Lander ES, Getz G: Discovery and saturation analysis of cancer genes across 21 tumour types. Nature 2014, 505:495-501.

32. Tamborero D, Gonzalez-Perez A, Perez-Llamas C, Deu-Pons J, Kandoth C, Reimand J, Lawrence MS, Getz G, Bader GD, Ding L, Lopez-Bigas N: Comprehensive identification of mutational cancer driver genes across 12 tumor types. Sci Rep 2013, 3:2650

33. Yang Q, Ota K, Tian Y, Kumar A, Wada J, Kashihara N, Wallner E, Kanwar YS: Cloning of rat fibrillin-2 cDNA and its role in branching morphogenesis of embryonic lung. Dev Biol 1999, 212:229-242.

34. Futreal PA, Coin L, Marshall M, Down T, Hubbard T, Wooster R, Rahman N, Stratton MR: A census of human cancer genes. Nat Rev Cancer 2004 , 4:177-183.

35. Karin M: Nuclear factor-kappaB in cancer development and progression. Nature 2006, 441:431-436.

36. Lee DF, Kuo HP, Chen CT, Hsu JM, Chou CK, Wei Y, Sun HL, Li LY, Ping B, Huang WC, He X, Hung J-Y, Lai C-C, Ding Q, Su J-L, Yang J-Y, Sahin AA, Hortobagyi GN, Tsai F-J, Tsai C-H, Hung M-C: IKK beta suppression of TSC1 links inflammation and tumor angiogenesis via the mTOR pathway. Cell 2007, 130:440-455.

37. Mosquera JM, Beltran H, Park K, MacDonald TY, Robinson BD, Tagawa ST, Perner S, Bismar TA, Erbersdobler A, Dhir R, Nelson JB, Nanus DM, Rubin MA Concurrent AURKA and MYCN gene amplifications are harbingers of lethal treatment-related neuroendocrine prostate cancer. Neoplasia 2013, 15:1-IN4.

38. Carreira S, Romanel A, Goodall J, Grist E, Ferraldeschi R, Miranda S, Prandi D, Lorente D, Frenel J-S, Pezaro C, Omlin A, Rodriques DN, Flohr P, Tunariu N, de Bono JS, Demichelis F, Attard G: Tumor clone dynamics in lethal prostate cancer. Sci Transl Med 2014, in press.

39. Chiang DY, Getz G, Jaffe DB, O'Kelly MJ, Zhao X, Carter SL, Russ C, Nusbaum C, Meyerson M, Lander ES: High-resolution mapping of copy-number alterations with massively parallel sequencing. Nat Methods 2009, 6:99-103.

40. Conrad DF, Pinto D, Redon R, Feuk L, Gokcumen O, Zhang Y, Aerts J, Andrews TD, Barnes C, Campbell P, Fitzgerald T, Hu M, Ihm CH, Kristiansson K, Macarthur DG, Macdonald JR, Onyiah I, Pang AWC, Robson S, Stirrups K, Valsesia A, Walter K, Wei J, Tyler-Smith C, Carter NP, Lee C, Scherer SW, Hurles 
ME: Origins and functional impact of copy number variation in the human genome. Nature 2010, 464:704-712.

41. Berger MF, Lawrence MS, Demichelis F, Drier Y, Cibulskis K, Sivachenko AY, Sboner A, Esgueva R, Pflueger D, Sougnez C, Onofrio R, Carter SL, Park K, Habegger L, Ambrogio L, Fennell T, Parkin M, Saksena G, Voet D, Ramos AH, Pugh TJ, Wilkinson J, Fisher S, Winckler W, Mahan S, Ardlie K, Baldwin J, Simons JW, Kitabayashi N, MacDonald TY, et al: The genomic complexity of primary human prostate cancer. Nature 2011, 470:214-220.

42. Demichelis F, Setlur SR, Beroukhim R, Perner S, Korbel JO, Lafargue CJ, Pflueger D, Pina C, Hofer MD, Sboner A, Svensson MA, Rickman DS, Urban A, Snyder M, Meyerson M, Lee C, Gerstein MB, Kuefer R, Rubin MA: Distinct genomic aberrations associated with ERG rearranged prostate cancer. Genes Chromosomes Cancer 2009, 48:366-380.

43. Alkodsi A, Louhimo R, Hautaniemi S: Comparative analysis of methods for identifying somatic copy number alterations from deep sequencing data. Brief Bioinform 2014, [Epub ahead of print].

44. Degner JF, Marioni JC, Pai AA, Pickrell JK, Nkadori E, Gilad Y, Pritchard JK Effect of read-mapping biases on detecting allele-specific expression from RNA-sequencing data. Bioinformatics 2009, 25:3207-3212.

45. Chakravarti IM, Laha RG: Handbook of Methods of Applied Statistics. New York: John Wiley \& Sons; 1967.

46. Moore RE, Kearfott RB, Cloud MJ: Introduction to Interval Analysis. Philadelphia: Society for Industrial and Applied Mathematics; 2009

47. CLONET: CLONality Estimate in Tumors. [http://demichelislab.unitn.t//CLONET]

48. Bitbucket: CLONET. [https://bitbucket.org/deid00/clonet]

\section{Submit your next manuscript to BioMed Central and take full advantage of:}

- Convenient online submission

- Thorough peer review

- No space constraints or color figure charges

- Immediate publication on acceptance

- Inclusion in PubMed, CAS, Scopus and Google Scholar

- Research which is freely available for redistribution 